\title{
AGN in the GOODS Fields: Variability and Multiwavelength Detection
}

\author{
Vicki L. Sarajedini ${ }^{1}$, David C. Koo ${ }^{2}$, and Alison J. Klesman ${ }^{1}$ \\ ${ }^{1}$ University of Florida, Department of Astronomy \\ Gainesville, FL 32611, USA \\ Email: vicki, alichan@astro.ufl.edu \\ ${ }^{2} \mathrm{UCO} /$ Lick Observatory, University of California \\ Santa Cruz, CA 95064, USA \\ Email: koo@ucolick.org
}

\begin{abstract}
Active galactic nuclei can be identified in deep $H S T$ surveys using different selection techniques and multiwavelength data. We aim to produce a complete sample of AGN in the GOODS South and North fields by combining X-ray, optical and mid-IR selection criteria, including galaxies displaying nuclear optical variability.
\end{abstract}

Keywords. galaxies:active, galaxies:nuclei

Identifying AGN in deep surveys requires a multiwavelength, multi-technique approach. We identify intrinsically faint AGN in the GOODS fields via their nuclear optical variability and compare this selection to X-ray and mid-IR detection techniques. The high resolution achievable with $H S T$ allows for the detection of small flux changes in the nucleus by isolating the nuclear light within the non-varying host galaxy (Sarajedini et al. 2003, 2006). Eighty-five of 4174 galaxies (2\%) display significant nuclear variability over a 6 -month time period. About half of the variables are also X-ray and/or mid-IR detected AGN candidates (Alexander et al. 2003; Luo et al. 2008; Alonso-Herrero et al. 2006, Donley et al. 2008).

The variable AGN candidates span a wide range of absolute magnitudes, redshifts and colors. The faintest candidates $\left(M_{B} \approx-16 \mathrm{mag}\right)$ are generally variable but undetected in X-rays, indicating that variability is an important technique for identifying intrinsically faint AGN/host galaxies. The most luminous candidates are identified as both variable and X-ray sources. Spectroscopic surveys have identified $31 \%$ of the optical variables as BLAGN and $\sim 7 \%$ as NLAGN.

Future work includes investigating the nature of the optically variable populations, particularly the non-X-ray/IR detected variables. Variables will be confirmed with a longer baseline of two years which is available for part of the GOODS fields. We will also perform X-ray stacking to look for signs of weak X-ray emission and examine the host galaxy SEDs and morphologies.

\section{References}

Alexander, D. M., et al. 2003, AJ, 126, 539

Alonso-Herrero, A. et al. 2006, ApJ, 640, 167

Donley, J. L., et al. 2007, ApJ, 660, 167

Luo, B., et al. 2008, ApJS, 179, 19

Sarajedini, V. L., et al. 2003, ApJ, 599, 173

Sarajedini, V. L., et al. 2006, ApJS, 166, 69 